\title{
Cognitive Scout Node for Communication in Disaster Scenarios
}

\author{
Rajesh K. Sharma, ${ }^{1}$ Anastasia Lavrenko, ${ }^{1}$ Dirk Kolb, ${ }^{2}$ and Reiner S. Thomä ${ }^{1}$ \\ ${ }^{1}$ International Graduate School on Mobile Communications, Ilmenau University of Technology, Helmholtzplatz 2, \\ 98684 Ilmenau, Germany \\ ${ }^{2}$ Reconnaissance Research \& Development (RRD) Division, MEDAV GmbH, Gräfenberger Straße 32-34, 91080 Uttenreuth, Germany
}

Correspondence should be addressed to Rajesh K. Sharma, rajesh-kumar.sharma@tu-ilmenau.de

Received 13 January 2012; Revised 26 April 2012; Accepted 22 May 2012

Academic Editor: Enrico Del Re

Copyright ( $) 2012$ Rajesh K. Sharma et al. This is an open access article distributed under the Creative Commons Attribution License, which permits unrestricted use, distribution, and reproduction in any medium, provided the original work is properly cited.

The cognitive radio (CR) concept has appeared as a promising technology to cope with the spectrum scarcity caused by increased spectrum demand due to the emergence of new applications. CR can be an appropriate mean to establish self-organization and situation awareness at the radio interface, which is highly desired to manage unexpected situations that may happen in a disaster scenario. The scout node proposed in this paper is an extended concept based on a powerful CR node in a heterogeneous nodes environment which takes a leading role for highly flexible, fast, and robust establishment of cooperative wireless links in a disaster situation. This node should have two components: one is a passive sensor unit that collects and stores the technical knowledge about the electromagnetic environment in a data processing unit so-called "radio environment map" in the form of a dynamically updated database, and other is an active transceiver unit which can automatically be configured either as a secondary node for opportunistic communication or as a cooperative base station or access point for primary network in emergency communications. Scout solution can be viable by taking advantage of the technologies used by existing radio surveillance systems in the context of CR.

\section{Introduction}

Communication has been an indispensable part of everyday life in the present days. Apart from making the general life better, modern communications should also be applicable for relief and support to the victims of exceptional adverse situations which include disaster scenarios like earthquakes, floods, cyclones, forest fires and terrorist attacks. Such scenarios impose new requirements on the communication systems. Some of the tasks of a cognitive radio network for emergency situations may be (1) to support specific service requests (higher traffic, coverage, localization, emergency messages, etc.), (2) to re-establish communications in a short time, and (3) to assist rescue forces communications and provide interoperability among them and also among rescue forces and public network.

One of the first tasks in disaster is to organize rescue operations in a quick and efficient manner which as well requires rescue forces to be provided with reliable and stable communication facilities. One of the common problems here is providing interoperability among rescue responders originally using different communication standards $[1,2]$. In terms of public communication systems, obvious problems in such scenarios are capacity overload with the resulting service denial and absence of coverage in some areas. Whereas the communication capabilities are in higher demand both for rescue responders and public users, the situation gets worse since the communication infrastructure may be fully or partially destroyed. Repairing the original network in a conventional way is time consuming and is not a correct measure in an exceptional situation. In such scenarios actual needs and requirements for communications can vary significantly depending on the scale of the disaster, place, and time elapsed since the beginning of the event. Therefore, a flexible and intelligent communication system which is aware of the situation and gets self-organized and adapted to the current operational demands is highly beneficial to deal with an unpredictable and time-varying situation. Naturally, 
cognitive radio (CR) capabilities seem to be highly potential for these purposes.

Cognitive radio (CR) has been considered as a technology for increasing spectral efficiency in wireless communications systems, by having sophisticated radios that can sense and take advantage of spectral opportunities [3]. Unlicensed CR users adaptively adjust radio parameters to the network environment, resulting in improved spectral efficiency. Cognitive radios (referred to as secondary users) may temporarily use spectrum as long as they do not interfere with primary users (PUs) that own the license to that spectrum. Although CR is often considered solely in terms of the use of temporary "white spaces" in the given frequency range detected by a secondary system, its capabilities can be useful for many other applications. Recently, several additional applications of CR have been investigated. For example, in [1], the application of CR for public safety along with other emerging applications has been discussed. The authors have also raised the related standardization issues for a CR technology to support such emerging applications. In [2], CR has been considered as an appropriate solution to the problems of public safety and emergency case communications, especially those related to interoperability issues.

If we consider exploiting $\mathrm{CR}$ in the disaster situation we need to address, however, a much broader and more exceptional problem area where the postdisaster unpredictable situation and its solution is needed to be taken into account. This includes analysis of the situation and reaction according to the current needs and priorities. Different levels of possible support should be envisaged: from providing additional services for local groups of users to establishing cooperation between the secondary and the primary system $[4,5]$. This results in a very broad range of requirements for the $\mathrm{CR}$ node. Therefore, the secondary node must be equipped with strong cognitive abilities to explore the situation, identify the available resources, and act according to the current need.

To utilize CR capabilities efficiently for an adequate and timely assistance in disaster situation, there is a need for obtaining relevant information on the service or system which requires support. For instance, to provide interoperability to the various emergency responders information on their modes of operation must be available which is not always a case especially in the presence of the forces subordinate to the different departments. In case of partial damage of the existing system infrastructure, there might be a need of support for its re-establishment which as well requires information on the system's operational parameters, capabilities, and current needs which demands increased cost and complexity. Here, the advantage can be taken from the current developments in the radio surveillance systems which basically solve similar problems but for different goals. Although the increased cost and complexity for advanced capabilities may not be justifiable for all of the secondary nodes, this can be justifiable for a single powerful node in the network which can assist other nodes for the improved performance.

In this paper, we propose a concept of a powerful cognitive node having extended capabilities which coexists in a network, where heterogeneous primary as well as secondary cognitive radios are in operation. This proposed node is different from other nodes in its features and missions. Firstly, it has more advanced sensing, signal processing capabilities, and additional flexibility in terms of mobility which are not available in normal cognitive nodes. Secondly, in the case of exceptional situation it gets self-organized in the system which needs support and provides some emergency services or reinforce affected services. Thirdly, during normal operation scenario it works as a secondary user (SU) in CR system making the cognitive communication more efficient and reliable avoiding interference to the primary system. The requirements of such node, its operating modes, its application scenarios, and research components for its design and development are discussed in this paper. The proposed system is found to have remarkable similarities with radio surveillance system; therefore, it can take the advantage of the later in several aspects. Note that the principal goal of such a node is an altruistic (cooperative) support in the disaster scenario rather than spectrum efficiency and throughput as it is considered in normal situations. Due to its foremost function of reconnaissance and observation in the radio environment, this node will be termed "scout" in this paper.

The remainder of the paper is organized as follows. Section 2 provides the general concept overview of the scout including its applications, operating scenarios and modes, and requirements. Section 3 discusses several issues and solutions for the most important task, the resource estimation and awareness, of the scout. Scout design and implementation issues are discussed in Section 4. Section 5 discusses briefly the current developments and operational issues in radio surveillance system from which scout can take advantage in terms of signal intelligence for its viability. Finally, the concluding remarks are given in Section 6.

\section{Scout System Concept}

Since one of the main aims of the cognitive radio node with extended capabilities is scouting-related radio resources, it is proposed here to call it "scout." The resources consist of radio spectrum resources that may be available in several dimensions like frequency, time, code, space, direction, polarization as well as the radio network infrastructure. The scout needs global information about radio resources for centralized decision to be taken by it and to act globally in the network. Some of the important global information related to radio resources include information about the primary systems and their modes of operation, unused frequency bands, the spatial and temporal statistics of its use, the distribution of the available nodes of the primary and secondary (CR) network, propagation characteristics in the primary and secondary network, and so forth. Although the need for knowing so many details about the primary system is unusual form the viewpoint of conventional (opportunistic) CR, it is important for scout since the "cooperative CR" vision is to be addressed by it. All the collected information must be stored in a database which in the literature is commonly referred to as a radio environment map (REM) [6]. 


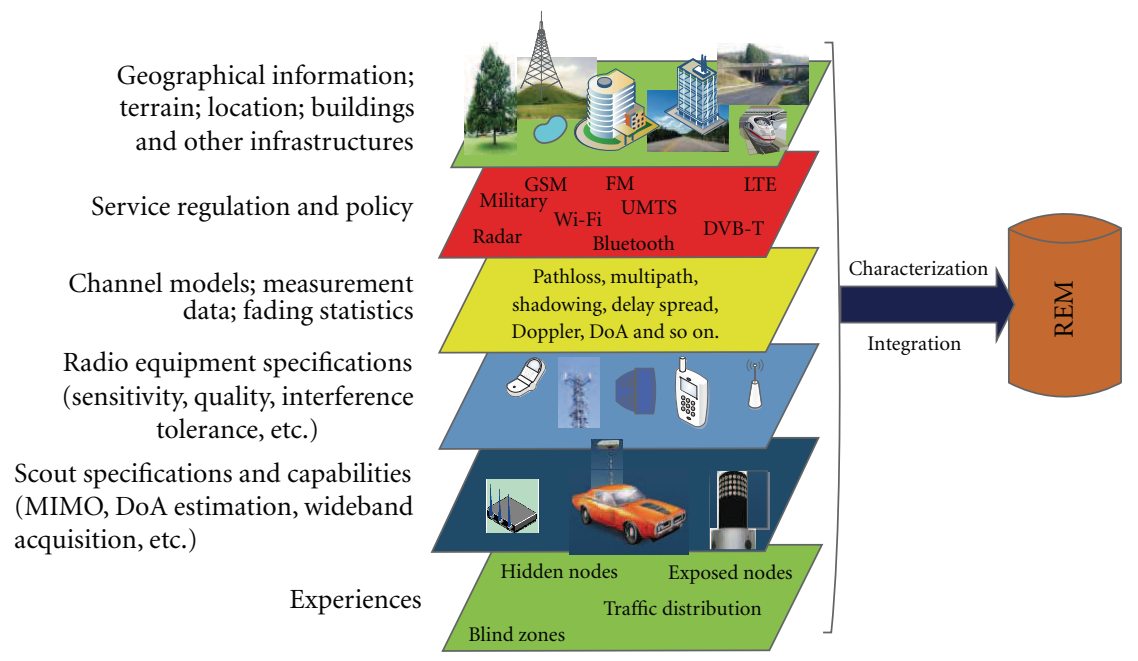

FIGURE 1: A REM obtained by integrating various databases (modified from [6]).

Radio environment map (REM) is an abstraction of realworld radio scenarios. It is an integrated spatiotemporal database which characterizes the radio environment of cognitive radios in multiple domains. It can be exploited to support cognitive functionality of the user equipment, even if the subscriber unit is relatively simple [6]. The illustration of how a REM is obtained from various information sources is shown in Figure 1. REM is assigned a central role in scout because different classes of collected sensor data (e.g., from the spectrum analysis and the physical layer analysis) and a priori knowledge are kept updated in it. It means that it consists of static and dynamic as well as temporally and spatially varying information in a well-managed form.

The cognitive engine is the brain of the CR system which executes a set of nested loops constituting a cognition cycle, drawing on experience and stored knowledge in REM to optimize a set of user-chosen quality-of-service measures [7]. Naturally, the scout must also be equipped with a more powerful cognitive engine than other CR nodes because it may need higher processing power for sensor signal data reduction or compression using relatively large database from the REM as well as real observation during its operation. Since it should be a more strategic databasesupported planning tool having situation awareness, it is something more than a simple cognitive engine and we can better term it as "cognitive planning tool."

The scout needs to take cognitive decision for its "act" phase which may be either a cooperation to the primary system or secondary communication according to its mission plan. Based on the sensing data, the a priori knowledge to the environment, the situation based on REM analysis, and agreed requirements the cognitive planning tool of the mobile scout provides the local decision as well as the decision based on the global (network-based) data processing in the heterogeneous nodes environment. Cognitive planning tool, which employs artificial intelligent (AI) techniques for cognitive mission control [8], has well defined cognitive control and decision rules, with the prioritization, classification, and consideration of various application requirements (disaster or mass event scenarios, etc.). It implements the duties of a mission control for the scout and also interacts with sensing and detection unit for learning.

During the operation of the network, the tasks (mission) of the scout could change and adapt according to the network status. While its first mission will be dominated by the simple exploration of the environment for supporting network establishment, the scout in later stages can switch to either altruistic or opportunistic communication mode in the network operation. A simple functional architecture of scout is shown in Figure 2(a), which is also expressed in the form of a scout cycle as shown in Figure 2(b) derived from a classical cognitive radio cycle [3].

Since scouts are envisaged to be powerful and versatile nodes working in a partially destroyed infrastructure primary network as well as a centralized/distributed secondary network, they should be able to estimate the resource, support the primary communication as an altruistic node in the form of relay nodes as well as communicate as secondary cognitive nodes in an opportunistic manner. The operation of the scout consists of two parts: one is the "observation and awareness" which is the task of collecting information from radio environment and storing it in REM. This can be also termed the scout mode of operation. The another operation is the "planning, deciding, and acting" by the scout which further results in two different roles to be played by it, based on which this operation can be classified into two modes: (1) altruistic support for primary communication as a relay node and (2) secondary opportunistic communication. These two operating modes can be seen in Figures 3(a) and $3(\mathrm{~b})$, respectively. It is apparent that the node requires to possess high level of reconfigurability in order to be able to operate in both of these modes.

In the altruistic communication mode, scout communicates to the primary network based on the collected information by it. It discovers the network status and 


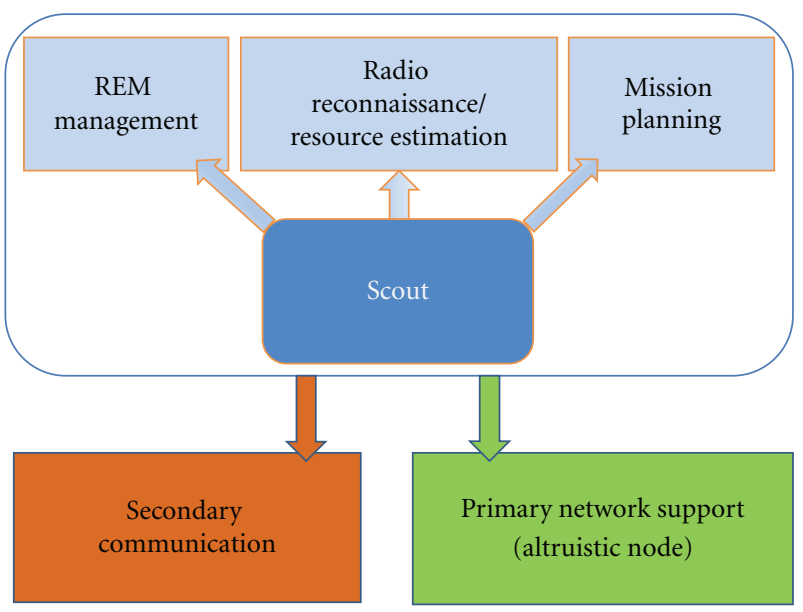

(a)

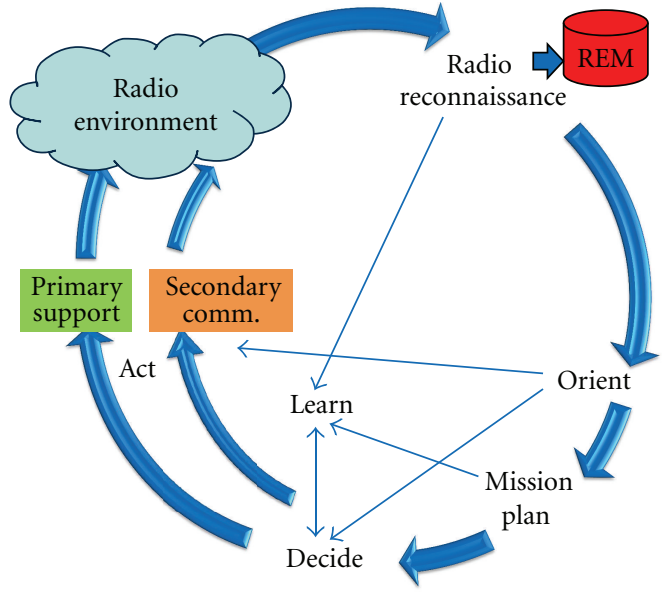

(b)

Figure 2: (a) A functional architecture of the scout and (b) a simple scout cycle of operation.
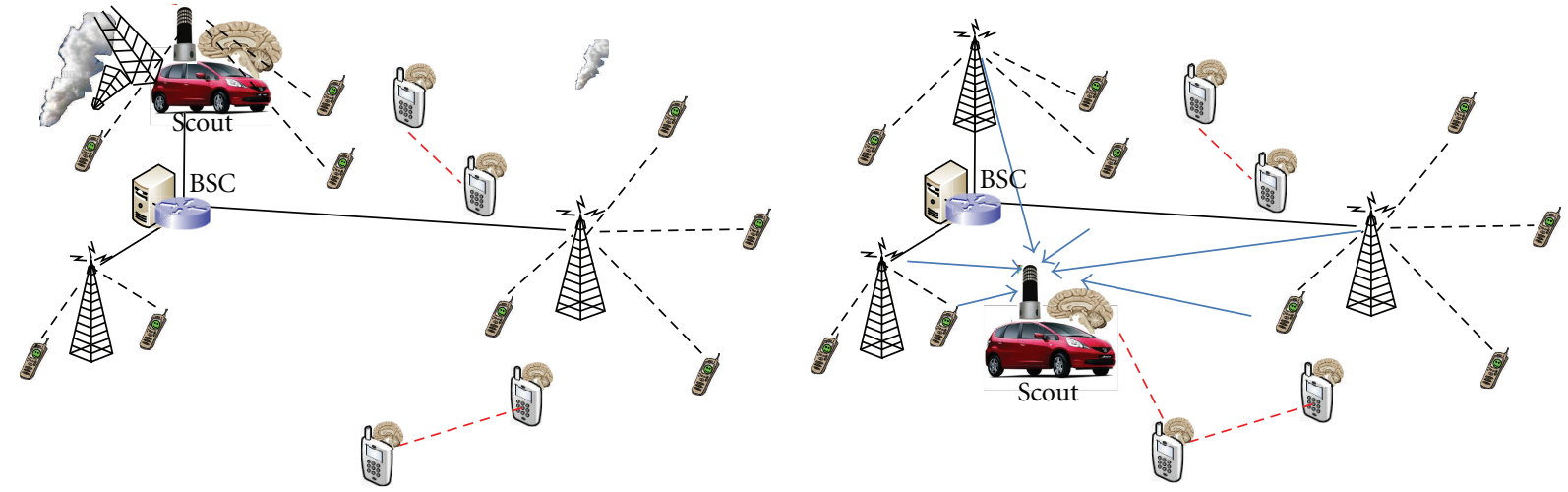

BSC: base station controller of primary network

$$
\begin{aligned}
& \text { BSC: base station controller } \\
& \text { of primary network } \\
& -- \text { Primary communication } \quad \text {--- Secondary communication } \\
& \longrightarrow \text { Sensing }
\end{aligned}
$$

- - - Primary communication

- - - Secondary communication

(a)

(b)

FIgURE 3: Scout in (a) altruistic primary communication support mode and (b) opportunistic secondary communication mode.

identifies the location of the failure. It gets self-organized itself in the primary network as a communication node and, even possibly, replaces the failed node and takes its role. This mode of operation requires signalling and synchronization issues to be resolved, and it poses highly sophisticated system requirements.

In the secondary opportunistic communication mode, the scout behaves simply as a CR node, but due to its extended capabilities it can contribute significantly more than other nodes for filling the REM whose information can be provided to other CR nodes in the network. Thus, it can support SU CR nodes which may have very limited sensing capabilities by giving valuable advice for radio resource access.

\section{Resource Estimation Capabilities of Scout}

Resource estimation is the first and the most important part of the scout task. Since the very exceptional operational conditions are considered, all the available equipment capabilities should be used in order to achieve the most robust and reliable estimation to ensure that resources would be fully and efficiently exploited in all the possible dimensions like spectrum, direction, location, and time. There may be different requirements for estimation depending upon the action to be taken by the scout. If the PU is observed for the purpose of secondary communication, the identification of white spaces may be sufficient. If the purpose of estimation is to help the victim users in primary system, the scout has 


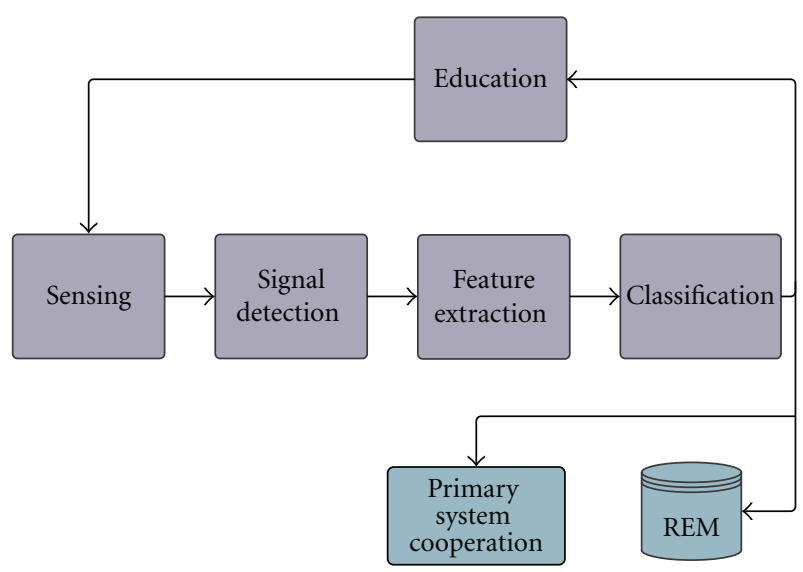

Figure 4: PHY mode identification and classification work-flow.

to find out what the primary users need and what physical layer (PHY) signalling they can accept. PU PHY mode identification and classification (from finite sets) is, therefore, essentially required especially for the second purpose. This includes detection of primary and/or secondary users and identification of their operational parameters. Here, one of the core tasks to perform is signal classification which could be implemented on the basis of pattern recognition theory using specific signal features of the considered communication standard. Features could characterize frequency domain like central frequency, bandwidth of a signal, frequency hopping pattern and/or time domain like waveform, and signal duration. A simple block diagram showing the workflow for PHY mode identification is shown in Figure 4.

Another possible estimation object is temporary statistics of primary and/or secondary users. To make the sensing and identification easy and reliable for the future events, the estimation of PU and SU statistics and storing the data in REM is important. It could involve time, frequency, and space domains as well as primary network state estimation, traffic estimation, and so forth. Some statistics is to be obtained from the measurements using the scout node itself. However, many background data including geographical information, terrain, buildings, service regulation, and so forth, provide additional valuable information for the estimation of primary user statistics. This information could be used as a base for prediction of the network status, choice of the secondary network PHY mode, and compiling higher level network protocols.

3.1. Advanced Spectrum Sensing. Some existing spectrum sensing techniques are matched filtering, waveform-based sensing [9], cyclostationary-based sensing [10-12], energy detection (ED) [13-16], autocorrelation-based sensing [17, 18], sample covariance-based detection [19], and cepstralbased detection [20,21].

For scout, it is mandatory to select the most reliable combination of features to handle the current radio scenarios successfully and to decrease the probability of errors. Talking about the combination of spectrum sensing together with REM, the question whether it is a good idea to use the

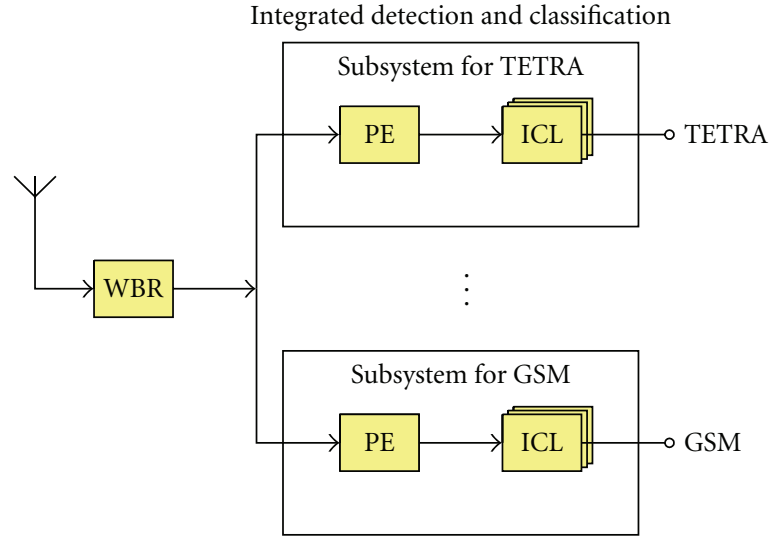

FIGURE 5: Processing chain with a common wideband receiver (WBR), transmission class-specific subsystems for pattern extraction (PE) and for integrated detection and classification (ICL).

information of the current radio environment to ease the processing always appears. If this information is used to support spectrum sensing, one should keep in mind that the radio scenario changes over time and location. Thus, it is mandatory that the spectrum sensing is updated automatically to fit to the current REM. Due to the fact that in some cases a priori information can be accessed and applied easily, however, in other cases the opposite happens as the current a priori information becomes irrelevant due to the new requirements. Nevertheless, for the operator of the scout, it is not obvious which kind of sensing technique is the best for a given radio scenario. So, the goal is to make the decision to choose the sensing features as automatically as possible.

If the scout starts in a new environment with noneducated sensing and PHY identification, it can later switch to educated sensing which not only makes the sensing easier but also enhance the sensing performance. This is actually the simple and straightforward application of cognitive principle which involves learning, cognition, and then educating other nodes.

In [21], a new sensing approach has been introduced where the main idea is to combine modern object detection techniques with new upcoming ideas from CR. By doing, this it was possible to combine different sensing approaches in such a way that a highly robust and real-time capable system for radio signal detection and classification emerged. The decision which sensing approach fits to the defined requirements was done automatically based on the current radio scenario or rather the current REM. Figure 5 shows the structure of the new system. It contains several subsystems for classification which are adapted to the radio standards of interest. This allows the operator of the scout to focus on updating the REM and not to spend too many efforts in trying to set up a spectrum sensing manually. In such case the sensing can be robust, fast, and reliable compared to the uneducated (blind) sensing. The neighbouring nodes with reduced sensing capabilities also can take advantage from the sensing decision of the scout. 


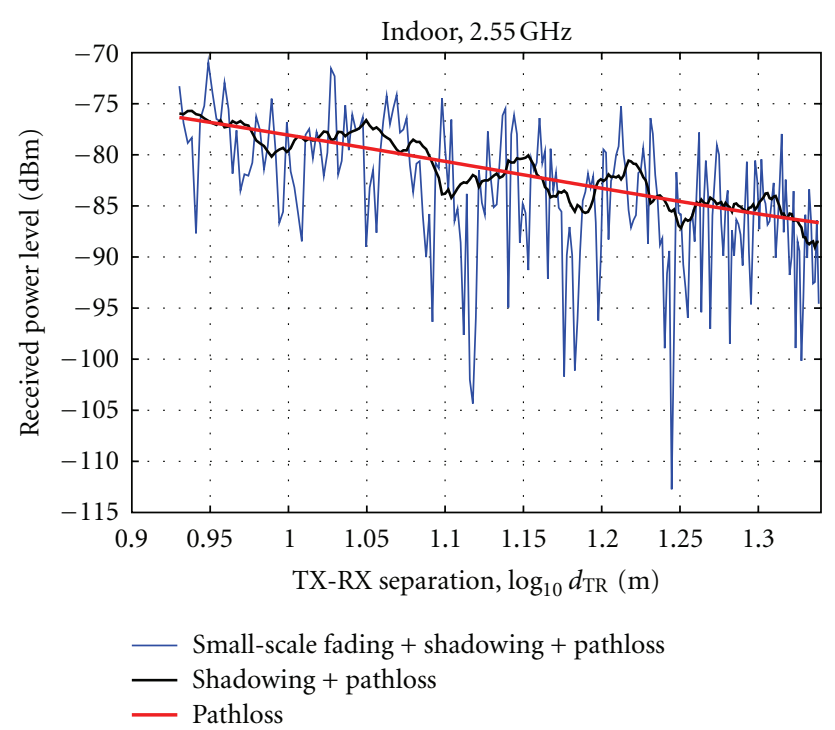

FIGURE 6: The received power in an indoor environment showing the effect of small-scale fading, shadowing, and pathloss.

3.2. Estimation of Channel Statistics. Since the sensing and estimation procedures all rely on the signals obtained from the sources in the environment, the reliability of the estimation from the received signal parameter depends upon the accuracy in the predicted channel parameters at the given location. The channel parameters of high importance are pathloss, small-scale fading parameters, and large-scale fading (shadowing) statistics. These parameters are illustrated in Figure 6 which is based on the real measurement in an indoor environment. Apart from these parameters delay spread based on power delay profile, Doppler spread, angle of arrival, and so forth in the given environment are the parameters to be estimated and stored in REM. Since exceptional disaster scenario is to be considered, the channel statistics and models used in the normal situation may not be applicable in this situation. Therefore, a realistic assumption of the channel statistics in disaster scenario is needed especially for initial attempts of estimation. Later, estimation of channel statistics based on current measurement results and the resource estimation and identification based on those new statistics will make the system highly reliable.

A main challenge in spectrum sensing and estimation arises due to hidden node problem. The hidden node problem can be caused by many factors including severe multipath fading or shadowing that secondary users observe while scanning PU transmissions. Here, the CR device causes unwanted interference to the PU (receiver) as the primary transmitter's signal could not be detected because of the positioning of devices in space. Since the hidden node problem arises due to the channel fading, the correct statistics of the fading in the radio environment helps to identify the hidden nodes. Channel statistics should be estimated by using PU and SU nodes as excitation signal sources.

It is important to note that fading goes along with other channel statistics, for example, angular spread and effective channel rank. So although knowledge about fading may be enough to explain the hidden node problem and to decide about channel availability for secondary communication, it will be not enough to assess the antennas influence and decide the optimum transmission mode including MIMO multiplexing versus diversity, beamforming, coding, and so forth. The small-as well as large-scale statistics are essential for cognitive link adaptation. In distributed and heterogeneous network, a cooperative link statistics are needed for the effective link and relay node implementation. For fulfilling these requirements, we should know more parameters and statistics related to the channel for the scout operation.

3.3. Tracking and Data Fusion. To use the information potential of the scout system in various decision tasks, the continuously collected data must not overwhelm the system. Instead, the data are to be condensed (fused) in such a way that high-quality information results serving as a basis for decision support in particular applications and on all levels of hierarchy [22].

Data fusion can also help to combine heterogeneous information having different types, sources, qualities, and so forth. It can help weight the quality and importance of information and help to handle incomplete and unreliable information. Data fusion is a very well-known method for the improvement of sensing performance. There might be different levels or layers of data fusion: from decision level that combines measured values(sensor decisions) [23] up to level where mostly data are retrieved, categorized, combined, and so forth [24].

Knowledge-based systems (KBS) can interpret the fusion results by considering and analysing issues such as the context in which the data are observed, the relationship among observed entitles, hierarchical groupings of targets or objects, and predictions of future actions of targets or entities [24]. Key issues for developing such a system include the creation of the knowledge base. Since the scout is a cognitive node equipped with a dynamically updated database (REM) and cognitive mission control unit, it can perform the knowledge-based data fusion for the better performance.

Fusion of sensor data may be utilized in different cases: data produced at different instants of time (i.e., target tracking), data being collected from different sensor sources, data with background information on the sensor performance as well as data with nonsensor context information [22]. Since scout is a single node, it can perform data fusion based on the information it collects at different instants of its movement (different locations) with a proper source tracking. If distributed nodes provide their local sensing results to the scout, it can also perform decision fusion based on the sensing results of the distributed nodes and its own sequential observations. And, as a consequence, the scout can plan its mission, which includes not only the choice of the track, to maximize its performance.

3.4. Information Compression (Compressive Sensing). It is a known fact from the success of lossy compression that most of the data we acquire are not important and do not cause the 


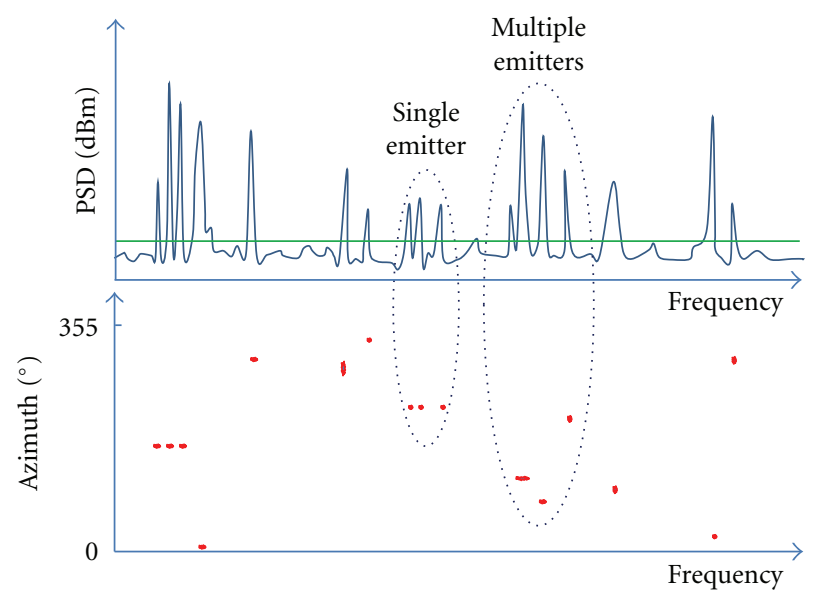

Figure 7: An example of data fusion between spectral and DoA information.

loss in information when they are discarded. If the sensing is performed just to acquire the important information, it makes the system efficient in the management of data. Practically, that might be the only possibility to handle the sensing process and to handle the huge amount of data.

There has been a significant research in the field of compressed sensing in the last few years. Compressed data acquisition protocols directly acquiring just the important information about the compressible signals have been proposed [25]. This sensing principle might enhance the sensing performance dramatically with reduced measurement time, reduced sampling rates, or reduced analog-to-digital (ADC) converter resources requirements [25].

A compressive sensing method capturing and representing compressible signals at a rate significantly below the Nyquist rate has been proposed in [26]. This method employs nonadaptive linear projections that preserve the structure of the signal. The signal is reconstructed from these projections using an optimization process. In [27], a Bayesian formalism has been employed for estimating the underlying signal based on compressive-sensing measurements. This framework has been utilized for CR primary user detection in [28], achieving the sampling reduction advantage with significantly less computational complexity.

Information compression using compressed sensing can play a significant role in scout performance by making the sensing more efficient and effective, whereas the REM remains efficient by storing compressed sensing data. Compression seems to be a solution to better exploit the limited hardware capabilities in terms of spectral coverage and speed, and also in terms of the required capacity of the data link to the fusion center.

\section{Scout Design and Implementation Issues}

The first task of the scout is to explore the spectral environment. The node should be compact enough for field operations on a mobile platform, for instance, a car.
Accordingly, the requirements include the selection of appropriate algorithms for signal analysis and signal estimation, appropriate platforms and approaches for implementation procedures. The appropriate methods of information fusion and database programming are to be used to establish the REM.

The important requirements in the implementation of scout include coherent multichannel receivers with wideband fast sensing capabilities. The antenna and bandwidth requirements are some of the key issues to be considered. Quality of service (QoS) and security issues are also important. Some of these issues are discussed here.

4.1. Antenna Requirements. From implementation point of view one of the important system parts required to perform estimation tasks discussed in Section 3 is wideband and/or tunable directional antennas which can gather information about spectrum resource in different directions for the given geographic location. Antenna arrays having direction-ofarrival (DoA) estimation capability makes the scout much more powerful than having a single antenna for both altruistic and opportunistic modes of operation. Direction finding is important for scout because it provides a bearing for a detected signal allowing to focus on a target area rather than random detection under the unknown resource distribution. It may be considered as a prerequisite for localization of a node. It makes possibility of tracking the target and allows intelligence fusion with other sensor data. By jointly exploiting spectrum and azimuth information, a more reliable automated emitter detection in dense scenarios becomes possible. For example, the spectral information combined with DoA information can distinguish between single emitter and multiple emitters along with their directions over a given frequency band as illustrated in Figure 7. It also provides SNR gain for the detection of weak signals. Separation of multiple stations for certain signal types is possible by direction finding. Additionally, high-resolution direction finding algorithms allow separation of multiple cochannel signals in the radio environment. Location and direction finding methods have been studied for long time $[29,30]$; however robust, fast, simple, and highly accurate algorithms are still interesting topics for today's research [31].

Although both beamforming and high-resolution DoA estimation are important for scout, they are different from each other. The beamforming gain in the former is strictly coupled to the size of the array. The latter can achieve high resolution performance in case of high SNR and precise antenna calibration. The requirements (and pitfalls) are described in [32].

The DoA estimation is possible with wideband array capability which makes scout more expensive and heavy. DoA estimation makes possibility of exploiting spatial dimension (direction) as an additional radio resource. Larger bandwidth, big antenna arrays, and multichannel receivers are needed for this purpose, which is a considerable effort in terms of cost and complexity. Adaptive beamforming [33], which requires also antenna arrays can be used by the node to 
control and avoid interference so that coexistence of primary and secondary network can be possible. Opportunistic antenna selection using orthogonal radiation patterns and/or polarization can be possible with small antenna arrays, but they have limited direction finding capability. Although such wideband array equipment may cost more than a base station today, there have already been many efforts to get cheaper equipment in radio surveillance community with a fast technological progress. Since the scout concept is for tomorrow's technology, its use in exceptional situations will be certainly feasible both technically and economically.

4.2. Frequency and Bandwidth Related Issues. Bandwidth requirement of antennas depends upon the specific task to be performed by the scout. During the sensing and reconnaissance phase, very wideband antenna or multiband antennas which consist of several antennas for the different frequency bands to scan the whole available bands may be highly desirable. However, for the communication phase, narrower band antenna to filter unwanted signals may be required. Reconfigurable antenna seems to be highly useful whose radiation pattern (direction; beamwidth) and bandwidth versus gain characteristics play an important role in the performance of the device.

The size of the antenna needed for scout operation depends upon the frequency band it is used for. Sometimes the antennas look large physically, but are small electrically. For example, if we talk about a wideband antenna in $60 \mathrm{GHz}$ band, the size will be small making the scout simple to implement. However, when lower $\mathrm{VHF} / \mathrm{UHF}$ bands are considered, physically large antennas will be required, and obtaining same bandwidth will be also more difficult. Designing wideband antennas in these bands need more weight and space for scout device.

In general, the lowest frequency of operation determines the antenna size. Sometimes we can use antennas that are electrically small, for example, for DoA resolution, which might work reasonably, but price that we have to pay is the reduced gain. This is a typical option for lower frequency. Fortunately, in this case the gain disadvantage can be partly compensated by lower free space attenuation. But there is always a compromise between gain, DoA resolution, and antenna array size which is influenced by platform size. Since the application of scout is broad, the use of narrowband and wideband as well as small and large antennas should be considered while designing the system.

4.3. Quality-of-Service Issues. For the application scenarios discussed in Section 2, different quality-of-service (QoS) requirements are to be satisfied by the scout node. In opportunistic communication mode, the scout working as a conventional CR must satisfy the interference constraint to the primary users. It must be able to serve for both real-time and best-effort traffic with the desired data rate and delay. Since there are different applications demanding large variation of capacity and delay requirements, cognitive engine should be able to make optimal transmission decision for the opportunistic communication.
The QoS requirements for altruistic communication mode are even more wider. For example, in one case, the scout acting as an altruistic node may need to establish a backbone connection to a local network of some users to get them connected to the public network. In the other extreme case, there may be a situation where it is necessary to reach a single user in a large distance. So, depending upon the user distribution and the radio environment there will be a much wider scope of QoS requirements to be fulfilled as compared to the regular networks. This requires high flexibility in the capability of scout node in terms of coverage, throughput, latency, reliability, and so forth. Since the QoS requirements may be interdependent, the choice of optimal parameters for the communication is important, which is to be performed by the cognitive planning tool. For example, choice of a frequency band will have influence on coverage (e.g., lower frequency can be used to reach distant users or to broadcast short messages, whereas higher frequencies are to be used to satisfy the high data rate demand in shorter distances). Beamforming versus multiplexing MIMO can be used to trade-off between coverage and capacity.

4.4. Security Issues. It is a well-known fact that there are malicious organizations that can attempt interception of the signals to and from the scout and make it to take incorrect decision in its mission. Any information flaw may result with harmful effect in the scene instead of fulfilling the desired goals. Therefore, it is crucial that the over the air information should be secure, which adds more challenge in the implementation. Although a detailed study on addressing the security issues on scout is important, it is not discussed here since it is beyond the scope of this paper.

\section{Current Developments in Signal Intelligence and Scout Viability}

Although the scout node discussed in this paper is a new concept, there are already technological achievements to serve a base for the scout development, especially in the radio surveillance applications. Also, the capabilities mentioned in Section 3 are expected to be achieved in the near future as many researches are focussed on these areas in cognitive radio perspectives.

Since to know what is on air has always been of strong interest, this noncivil market of radio surveillance can look back on a long development of several decades. A typical radio surveillance system consists of one or several wideband receivers, digital signal processing units, and some postprocessing systems. Dependent on the task of such a system, different capabilities, for example, radio signal detection and classification; direction or location finding, can be enabled. Ongoing developments show that especially the tactical systems for direction or location finding get smaller and consume less power which is ideal for vehiclebased missions. To fulfil a radio surveillance task, high flexibility of the applied system is necessary. It can happen that the mission details, for example, a priori information regarding occupied frequencies, radio services and networks, 


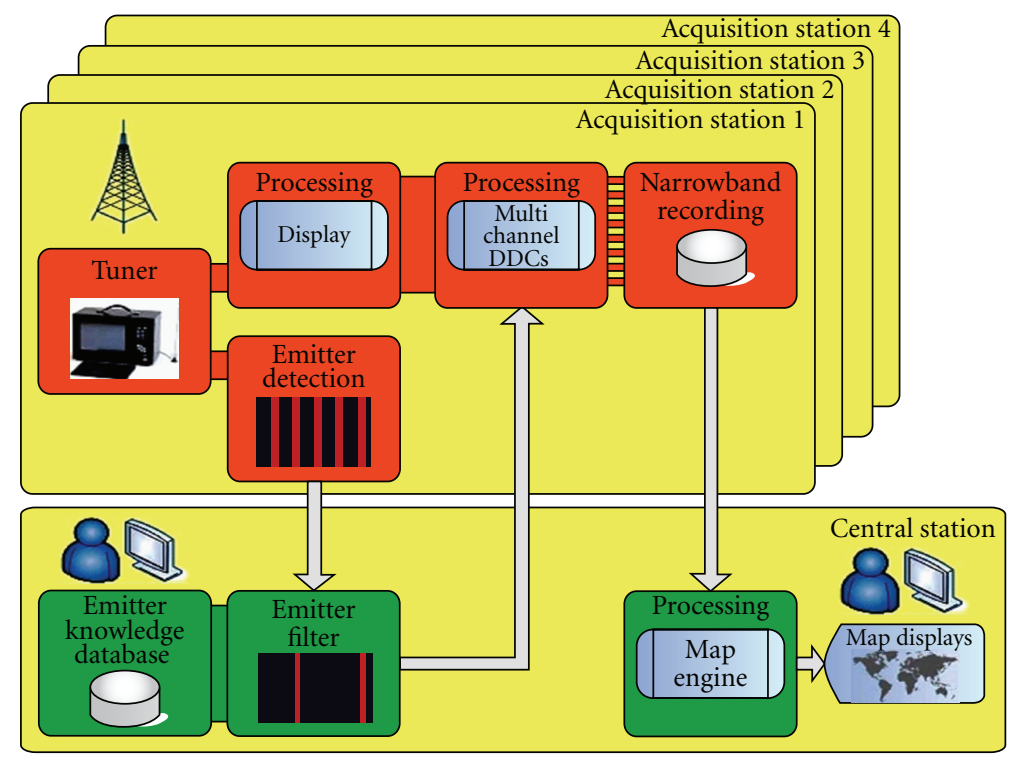

FIGURE 8: Multisensor setup of the new radio surveillance system developed by MEDAV.

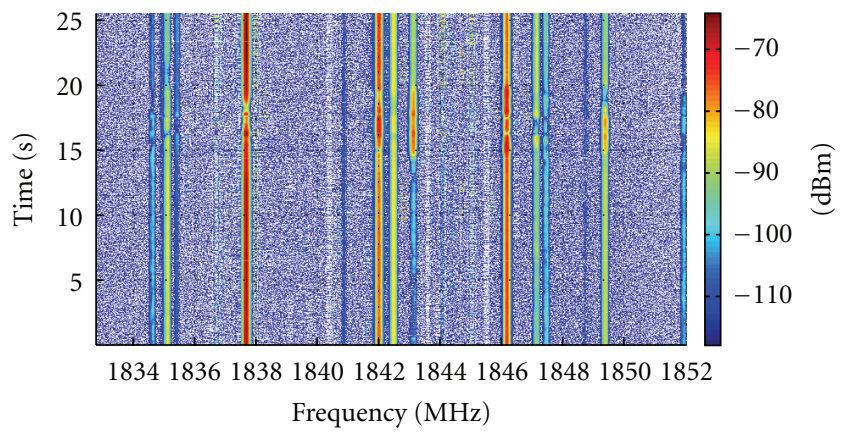

FIGURE 9: Spectrogram obtained by the wideband receiver IZT R3200 in GSM-1800 downlink.

and their assumed locations change over time, frequency, and especially over location. In case of a dislocation of a radio surveillance system, there is a high probability that new radio standards of interest appear. Thus, every stage of the processing chain, for example, the signal detection and classification, should be adaptable to new radio scenarios. It is obvious that the systems for radio surveillance are technically similar to the scout, which is proposed. In addition to the technical conformities, there are a lot of common aspects regarding the mission process itself. In sum, it would be a good idea to combine the knowledge gained from radio surveillance and the ideas of the research areas like cognitive radio in order to create smarter solutions.

As an example, a new system of MEDAV [34] for monitoring of fast changing radio scenarios contains tools for mission planning, sensor controlling, and real-time situation pictures. With this software it is possible to control and task not only the proposed direction finding (DF) system but also other sensor types. The modular concept allows to do most of the signal processing, for example, radio signal detection and classification, within the scout and to gather the resulting information in the fusion center to combine it with information coming from other sensors. The focus of the system is on mobile sensors with robust data links and low data rates between the sensor and the fusion center. Thus, it is a perfect basis for the proposed scout concept. Figure 8 shows the structure of a multisensor setup.

Keeping most of the signal processing and analysis in the software, antenna and radio front-end characteristics, however, still play a key role imposing the strongest restrictions on the overall sensing capabilities of scout. Since the main objective of scout is the assessment of the radio environment, one of the important aspects for its viability is existence of appropriate receivers. Currently, there are already some solutions available on the market which could be suitable for the scout design. For instance, digital wideband receiver R3200 developed by IZT GmbH [35] can cover frequency range from $9 \mathrm{kHz}$ to $18 \mathrm{GHz}$ with the maximum real-time instantaneous bandwidth of $19.2 \mathrm{MHz}$ for I/Q baseband data. As an example, a spectrogram which is obtained by this receiver in an indoor location is shown in Figure 9, which gives a clear picture of the instantaneous user activity in GSM-1800 downlink.

Since the radio surveillance systems for small-sized vehicular platform are already available in the market [34, $36,37]$, scout can take advantage from the technology used by these systems with the addition or enhancement of the capabilities in resource estimation as discussed in Section 3, including a full-fledged REM, and also addressing the issues mentioned in Section 4.

\section{Conclusion}

In this paper, we have discussed an extended concept based on cognitive radio which can be used as a secondary communication as well as a cooperative (altruistic) node 
for partially destroyed primary network infrastructure. The different requirements and implementation issues for such a node (scout) have been discussed. Since the required functionalities are advanced but still achievable in the present day of very high speed processors as well as the storage devices, a special cognitive node with such advanced features can be a highly useful and feasible system for the communication support to the victims in the exceptional situations including disasters and terrorist attacks.

\section{Acknowledgments}

This work has been carried out within the International Graduate School on Mobile Communications supported by the German Research Foundation (DFG) under the project "GRK 1487," and the Carl-Zeiss Foundation.

\section{References}

[1] J. Wang, M. Ghosh, and K. Challapali, "Emerging cognitive radio applications: a survey," IEEE Communications Magazine, vol. 49, no. 3, pp. 74-81, 2011.

[2] A. Gorcin and H. Arslan, "Public safety and emergency case communications: opportunities from the aspect of cognitive radio," in Proceedings of the 3rd IEEE Symposium on New Frontiers in Dynamic Spectrum Access Networks (DySPAN '08), pp. 1-10, October 2008.

[3] J. Mitola and G. Q. Maguire Jr., "Cognitive radio: making software radios more personal," IEEE Personal Communications, vol. 6, no. 4, pp. 13-18, 1999.

[4] Use Cases for Cognitive Applications in Public Safety Communications Systems-Volume 1: Review of the 7 July Bombing of the London Underground, Wireless Innovation Forum, 2007, http://groups.winnforum.org/d/do/1565.

[5] Use Cases for Cognitive Applications in Public Safety Communications Systems Volume 2, Chemical Plant Explosion Scenario, Wireless Innovation Forum, 2010, http://groups. winnforum.org/d/do/2325.

[6] Y. Zhao, J. H. Reed, S. Mao, and K. K. Bae, "Overhead analysis for radio environment mapenabled cognitive radio networks," in Proceedings of the 1st IEEE Workshop on Networking Technologies for Software Defined Radio Networks (SDR '06), pp. 18-25, September 2006.

[7] B. Le, T. W. Rondeau, and C. W. Bostian, "Cognitive radio realities," Wireless Communications and Mobile Computing, vol. 7, no. 9, Article ID 129497, pp. 1037-1048, 2007.

[8] A. He, K. K. Bae, T. R. Newman et al., "A survey of artificial intelligence for cognitive radios," IEEE Transactions on Vehicular Technology, vol. 59, no. 4, pp. 1578-1592, 2010.

[9] A. Sahai, R. Tandra, S. M. Mishra, and N. Hoven, "Fundamental design tradeoffs in cognitive radio systems," in Proceedings of the 1st International Workshop on Technology and Policy for Accessing Spectrum (TAPAS '06), August 2006.

[10] M. Öner and F. Jondral, "Cyclostationarity based air interface recognition for software radio systems," in Proceedings of the IEEE Radio and Wireless Conference (RAWCON '04), pp. 263266, September 2004.

[11] R. S. Roberts, W. A. Brown, and H. H. Loomis Jr., "Computationally efficient algorithms for cyclic spectral analysis," IEEE SP Magazine, vol. 8, no. 2, pp. 38-49, 1991.

[12] D. Cabric, "Addressing the feasibility of cognitive radios: using testbed implementation and experiments for exploration and demonstration," IEEE Signal Processing Magazine, vol. 25, no. 6, pp. 85-93, 2008.

[13] H. Urkowitz, "Energy detection of unknown deterministic signals," Proceedings of the IEEE, vol. 55, no. 4, pp. 523-531, 1967.

[14] V. I. Kostylev, "Energy detection of a signal with random amplitude," in Proceedings of the International Conference on Communications (ICC '02), pp. 1606-1610, May 2002.

[15] F. F. Digham, M. S. Alouini, and M. K. Simon, "On the energy detection of unknown signals over fading channels," in Proceedings of the International Conference on Communications (ICC '03), pp. 3575-3579, May 2003.

[16] A. Ghasemi and E. S. Sousa, "Asymptotic performance of collaborative spectrum sensing under correlated log-normal shadowing," IEEE Communications Letters, vol. 11, no. 1, pp. 34-36, 2007.

[17] R. K. Sharma and J. W. Wallace, "Improved spectrum sensing by utilizing signal autocorrelation," in Proceedings of the IEEE 69th Vehicular Technology Conference, pp. 1-5, Barcelona, Spain, April 2009.

[18] R. K. Sharma and J. W. Wallace, "Correlation-based sensing for cognitive radio networks: bounds and experimental assessment," IEEE Sensors Journal, vol. 11, no. 3, pp. 657-666, 2011.

[19] Y. Zeng and Y. C. Liang, "Spectrum-sensing algorithms for cognitive radio based on statistical covariances," IEEE Transactions on Vehicular Technology, vol. 58, no. 4, pp. 18041815, 2009.

[20] M. Li, V. Rozgić, G. Thatte et al., "Multimodal physical activity recognition by fusing temporal and cepstral information," IEEE Transactions on Neural Systems and Rehabilitation Engineering, vol. 18, no. 4, pp. 369-380, 2010.

[21] D. Kolb, U. Uebler, and E. N. Nöth, "A novel transmission scanner framework for real-time applications," in Proceedings of the RTO-MPIST-092- Military Communications and Networks. NATO Research and Technology Organisations, 2010.

[22] W. Koch, "On Bayesian tracking and data fusion: a tutorial introduction with examples," IEEE Aerospace and Electronic Systems Magazine, vol. 25, no. 7, pp. 29-51, 2010.

[23] Z. Chair and P. K. Varshney, "Optimal data fusion in multiple sensor detection systems," IEEE Transactions on Aerospace and Electronic Systems, vol. 22, no. 1, pp. 98-101, 1986.

[24] D. L. Hall and J. Llinas, "An introduction to multisensor data fusion," Proceedings of the IEEE, vol. 85, no. 1, pp. 6-23, 1997.

[25] D. L. Donoho, "Compressed sensing," IEEE Transactions on Information Theory, vol. 52, no. 4, pp. 1289-1306, 2006.

[26] R. G. Baraniuk, "Compressive sensing," IEEE Signal Processing Magazine, vol. 24, no. 4, pp. 118-121, 2007.

[27] S. Ji, Y. Xue, and L. Carin, "Bayesian compressive sensing," IEEE Transactions on Signal Processing, vol. 56, no. 6, pp. 23462356, 2008.

[28] S. Hong, "Multi-resolution bayesian compressive sensing for cognitive radio primary user detection," in Proceedings of the 53rd IEEE Global Communications Conference (GLOBECOM '10), pp. 1-6, December 2010.

[29] A. J. Berni, "Angle-of-arrival estimation using an adaptive antenna array," IEEE Transactions on Aerospace and Electronic Systems, vol. 11, no. 2, pp. 278-284, 1975.

[30] S. S. Reddi, "Multiple source location-a digital approach," IEEE Transactions on Aerospace and Electronic Systems, vol. 15, no. 1, pp. 95-105, 1979.

[31] S. D. Blunt, T. Chan, and K. Gerlach, "Robust DOA estimation: the reiterative superresolution (RISR) algorithm," IEEE Transactions on Aerospace and Electronic Systems, vol. 47, no. 1, pp. 332-346, 2011. 
[32] M. Landmann, M. K. Käske, and R. S. Thomä, "Impact of incomplete and inaccurate data models on high resolution parameter estimation in multidimensional channel sounding," IEEE Transactions on Antennas and Propagation, vol. 60, no. 2, pp. 557-573, 2012.

[33] R. M. Radaydeh and M.-S. Alouini, "Impact of co-channel interference on the performance of adaptive generalized transmit beamforming," IEEE Transactions on Wireless Communications, vol. 10, no. 8, pp. 2616-2629, 2011.

[34] Radio Monitoring and Surveillance Solutions, MEDAV, 2011, http://www.medav.de/.

[35] IZT R3200 Digital Wideband Receiver, IZT GmbH, 2012, http://www.izt-labs.de/en/products/kategorie/receivers/produkt/izt-r3200-1/.

[36] Radio Surveillance Overview, Synectics, 2011, http://www. synx.com/index.php/Products/radio-surveillance.html.

[37] Radio Surveillance and Intelligence, Morcom, 2011, http:// www.morcom.com/. 

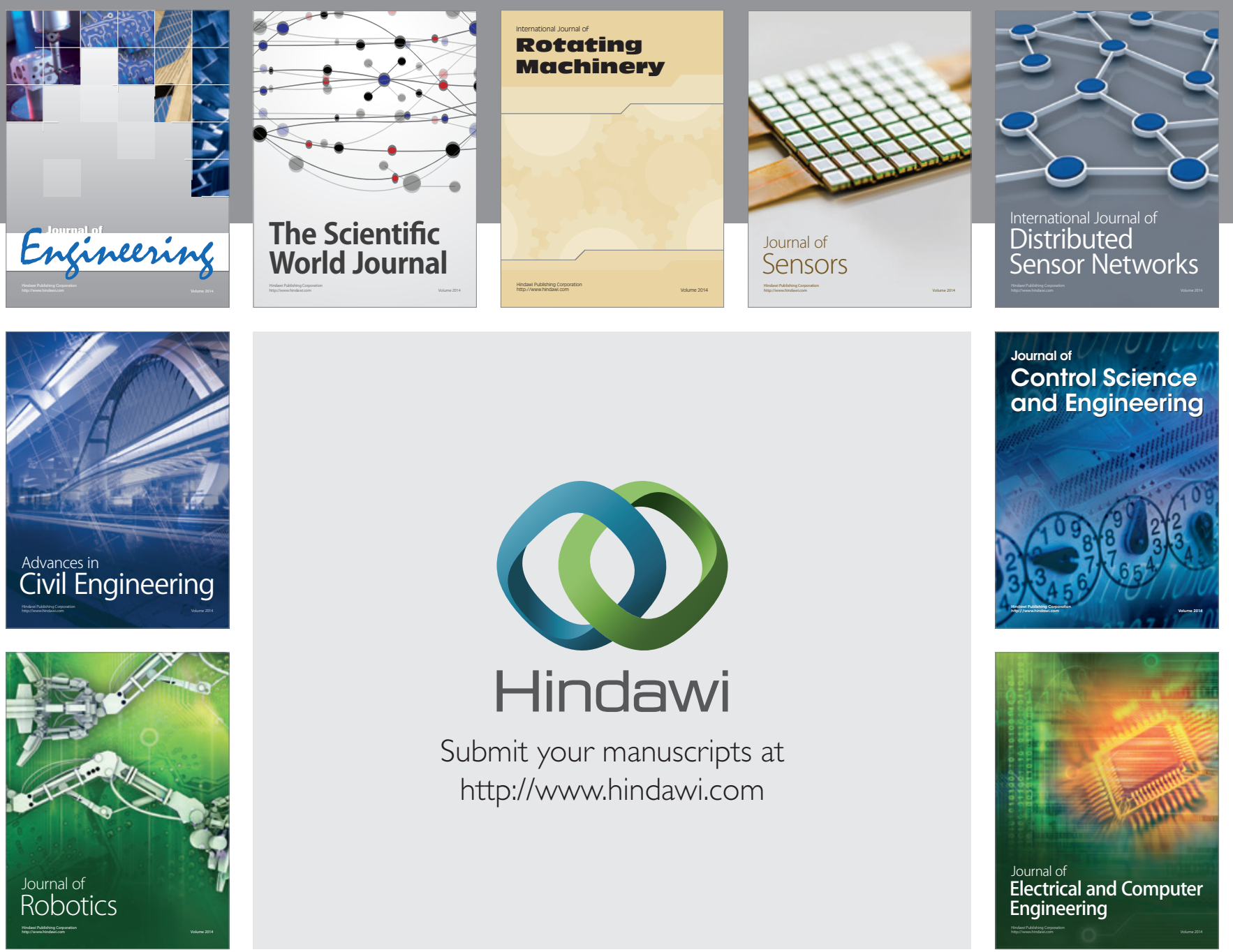

Submit your manuscripts at

http://www.hindawi.com
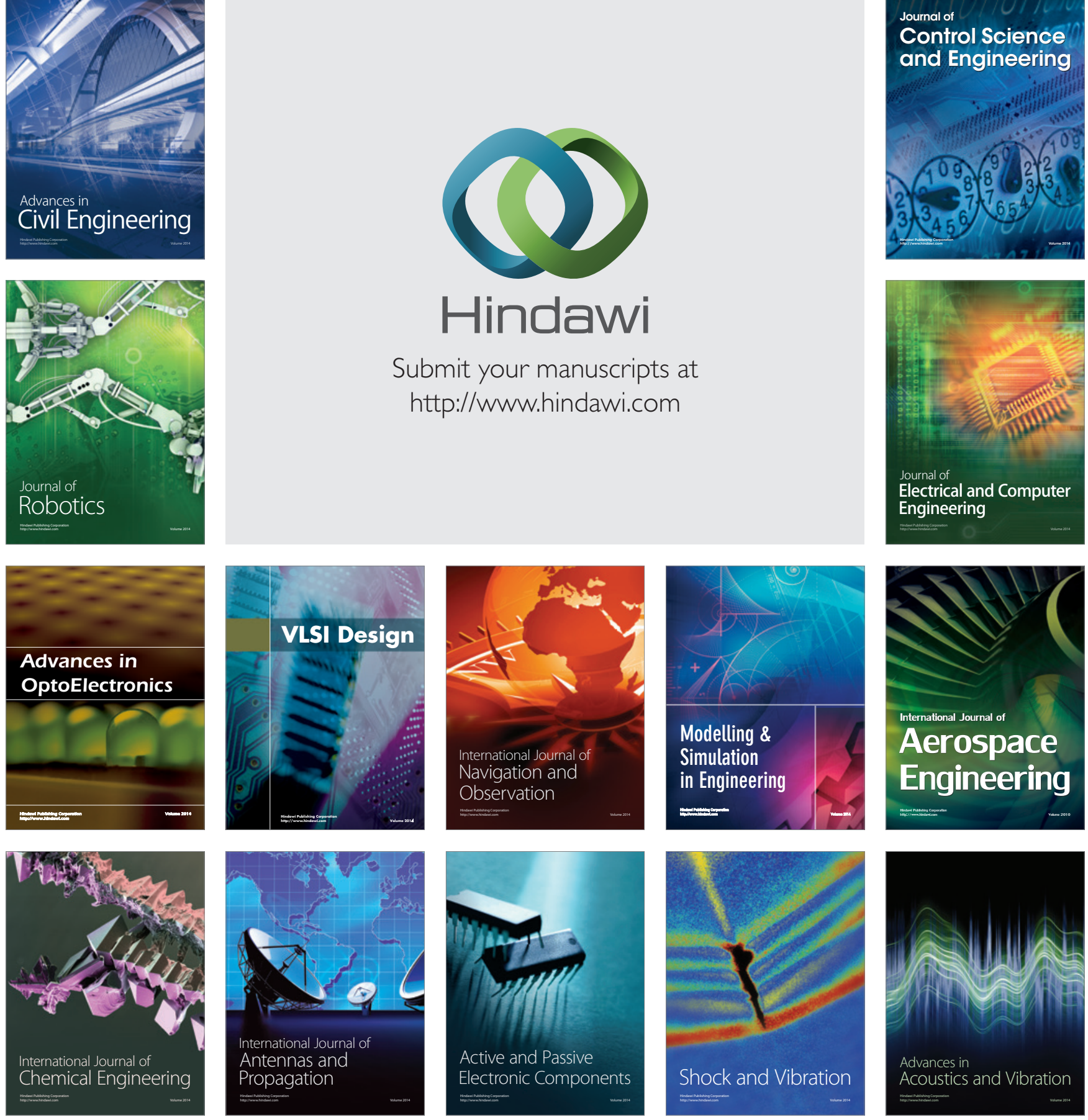